\title{
Extracts and essential oil of two medicinal plants as a candidate against urinary tract infection caused by multi-drug resistance $E$. coli
}

Mohammad Ahmadian

University of Guilan

Mohammad Javad Mehdipour Moghaddam ( $\nabla$ mj_mehdipour@guilan.ac.ir)

University of Guilan https://orcid.org/0000-0002-0392-065X

\section{Research}

Keywords: Extract, Essential oil, Resistance, Plant, E. coli

Posted Date: September 30th, 2020

DOI: https://doi.org/10.21203/rs.3.rs-81445/v1

License: (c) (i) This work is licensed under a Creative Commons Attribution 4.0 International License.

Read Full License 


\section{Abstract}

Background: The antimicrobial activity of ethanolic and aqueous extracts of the two plants, including Eryngium caucasicum Trautv (EtEc, AqEc) and Froriepia subpinnata (EtFs, AqFs), as well as the essential oil of Eryngium caucasicum (EoFs) on MDR E. coli spp. isolated from the urine samples were examined.

Methods: Microdilution and agar well diffusion methods were used to determine the minimum inhibitory concentration (MIC), minimum bactericidal concentration (MBC) and inhibition zone diameter of the extracts and essential oil. Reverse-phase High- performance liquid chromatography (RP-HPLC) and gas chromatography-mass spectrometry (GC-mass) were applied for phytochemical analyses of extracts and essential oil ingredients.

Results: The inhibitory rate based on MIC of AqEc, EtEc, AqEc, EtEc and EoEs on total isolates were 37.6\%, $43 \%, 31.2 \%, 40.9 \%$ and $53.8 \%$, respectively. The killing rate based on MBC of AqEc, EtEc, AqEc, EtEc and EoFs on total isolates were $15.1 \%, 20.4 \%, 12.9 \%, 24.7 \%$ and $34.4 \%$, respectively. EoFs, with the exception of cephalothin, chloramphenicol, pipiracillin and carbapenems, can be used as an alternative to 12 of the 18 antibiotics used $(P<0.01)$. Compounds with antimicrobial activity including quercetin, catechin, terpenes, alcohols and aromatic molecules in extracts and essential oils have been identified and measured, but chlorogenic acid polyphenol could not be identified in any of the plant products.

Conclusion: It seems that the extracts and essential oil studied have the potential to be used as native materials to treat urinary tract infections as well as food preservatives or other products.

\section{Background}

Escherichia coli is a major cause of extraintestinal, enteric and systemic infections in human and animal. Uropathogenic E. coli (UPEC), a member of the pathogenic extra-intestinal E. coli (ExPEC), is a major pathogen that causes urinary tract infections (UTIS). UPEC is one of the main causes of community and nosocomial acquired UTIs [1]. UTIs are among the most common infections of the bacteria from all over the world. It is estimated that 150 million UTIs occur worldwide annually. In the United States, the ExPEC strains are responsible for the deaths of 40,000 people and the cost of $\$ 2.6$ billion in medical care. Although infections can be treated, the emergence of multidrug-resistant (MDR) bacteria has led to defects in treatment and increased mortality and morbidity $[2,3]$.

Since antibiotic resistance in MDR strains is on the rise, it is inevitable to look for alternatives to antibiotics. According to a World Health Organization (WHO) survey, more than $80 \%$ of the world's population relies heavily on the use of traditional medicine plants to treat most infectious diseases. In the pharmaceutical industry a wide range of plant-produced secondary metabolites such as alkaloids, flavonoids, phenolic compounds, tannins, and steroids are used as bioactive compounds $[4,5,6]$. A number of scientific evidence has shown edible and medicinal plants have high potential to synthesize antimicrobials as defense mechanisms against microorganisms [7]. 
In this study, the antimicrobial effects of two plants from the Apiaceae family, including Eryngium caucasicum Trautv and Froriepia subpinnata L., were investigated. The family of Apiaceae (Umbelliferae) is large, with more than 3,000 species cultivated worldwide for food, such as vegetables, herbs, spices or medicinal use [8].

Genus Eryngium is the greatest and most complex genus in the family Apiaceae. In Iran, nine species of this genus have been found [9]. Some species, such as Eryngium maritimum, Eryngium foetidum, Eryngium campestre, and Eryngium planum were used as food or in folk medicine, both locally and worldwide $[10,11]$ and also some species of Eryngium are cultivated as a vegetable crop in Asia and Africa $[12,13]$. Valuable usage of Eryngium species are well known in traditional medicines in many countries. Aerial and root portions are used to treat infections, cancers, kidney stones, skin diseases, as well as to promote diuresis and control blood pressure $[14,15]$. Some recent bioactivity studies have proven traditional medical applications related to antioxidant activity, anti-inflammatory, anti-nociceptive, and haemolytic of this plant $[16,17]$. Compounds including terpenoids, triterpenoid saponins, flavonoids, coumarins, polystylenes, steroids and essential oils have also been reported in the genus Eryngium [9].

Eryngium caucasicum Trautv is a perennial herbaceous plant that is endemic to northern Iran and in these areas, especially in Gilan and Mazandaran provinces; mainly young leaves are collected wild and sold in local markets. It's called "Aweiyeh or Choochakh" locally. The leaves are used as food flavoring additive with anti-spoil features in Hyrcanian folklore nutrition traditions [18]. Phytochemical analysis shows the presence of compounds such as sequiterpenes, monoterpenes in the essential oil of Eryngium caucasicum Trautv $[17,19,20]$.

Froriepia is one of the Apiaceae genera, of which only one species called Froriepia subpinnata is found in Iran. Froriepia subpinnata (Ledeb.) Baill. was natively observed in Guilan, Mazandaran and Golestan provinces of Iran [21,22], but also grows widely in the northern, western and central regions of Iran and especially in cold-climate mountain areas. There are 150 species of Froriepia subpinnata in Eurasia and Africa and more than 16 in Europe [23]. The plant's pristine leaves are used as a vegetable for cooking and flavoring many local foods in most parts of north Iran. This plant has been used in traditional medicine as diuretic, sedative, antispasmodic agent [24]. Froriepia subpinnata contains antioxidant and antibacterial properties and essential oils of 39 species have been identified as anti-cancer compounds [25].

In this study it is intended to investigate the antimicrobial effects of ethanolic and aqueous extracts of Eryngium caucasicum Trautv and Froriepia subpinnata L. and also the essential oil of Eryngium caucasicum Trautv on clinical isolates of MDR E. coli, considering the various benefits of plant materials, including their antimicrobial activity.

\section{Methods}

\section{E. coli isolates}


Urine samples have been used to isolate $E$. coli isolates from patients with UTIs. Patient urine samples (138 samples including 31 male and 107 female specimens with mean age of 43 for male and 41 for female) were obtained in the early morning mid-stream using sterile, large mouthed glass bottles with tops of the screw cap between May to July 2015. Samples were stored in an icebox until laboratory analysis was done. Among sample collection and its analysis, it did not last for more than one hour. The standard bacteriological methods were used to cultivate, isolate and classify the strains. Urine samples were grown on the Nutrient, Blood, Eosin Methylene Blue (EMB) and MacConkey agar plates and incubated for $18-24 \mathrm{~h}$ at $37^{\circ} \mathrm{C}$. Urine culture was considered as positive, if it included $\geq 10^{5} \mathrm{cfu} / \mathrm{ml}$. Identification of $E$. coli strains from positive urine culture is performed using Gram staining, growth on the media and using biochemical tests [26]. The isolates were stored for further study at $-70^{\circ} \mathrm{C}$ in a Tryptic Soy Broth that contained 15\% glycerol.

\section{Antimicrobial susceptibility testing}

The disc diffusion method was used according to the Clinical and Laboratory Standards Institute [27] to determine the susceptibility of the isolates to antibiotics. The following antibiotic discs were used to perform this test. amoxicillin (AMX) $(25 \mu \mathrm{g})$, ampicillin (AMP) $(10 \mu \mathrm{g})$, oxacillin (OXA) $(5 \mu \mathrm{g})$, piperacillin (PIP) $(100 \mu \mathrm{g})$, amoxicillin-clavulanic acid (AMC) $(20 / 10 \mu \mathrm{g})$, fosfomycin (FOF) $(200 \mu \mathrm{g})$, tetracycline (TE) $(30 \mu \mathrm{g})$, chloramphenicol (CHL) $(30 \mu \mathrm{g})$, streptomycin (STR) $(10 \mu \mathrm{g})$, ciprofloxacin (CIP) $(5 \mu \mathrm{g})$, cephalothin (CEF) $(30 \mu \mathrm{g})$, cefazolin (CFZ) $(30 \mu \mathrm{g})$, cefuroxime (CXM) $(30 \mu \mathrm{g})$, cefixime (CFM) $(5 \mu \mathrm{g})$, ceftazidime (CAZ) $(30 \mu \mathrm{g})$, ceftriaxone (CRO) $(30 \mu \mathrm{g})$, cefotaxime (CTX) $(30 \mu \mathrm{g})$, cefepime (FEP) $(30 \mu \mathrm{g})$, imipenem (IMP) $(10 \mu \mathrm{g})$, meropenem (MEM) $(10 \mu \mathrm{g})$. E. coli ATCC 25922 and E. coli ATCC 35218 were used as standard strains for controlling the performance of the antimicrobial agents applied. MDR is described as having three or more antibiotics resistance.

\section{Plant extraction preparation}

Leaves from Eryngium caucasicum Trautv (APE53281), and Froriepia subpinnata (APF54075) were collected from the Lahijan and Rasht counties, in Guilan province, northern Iran, in August 2015. Plant identification was performed by Dr Davood Bakhshi and voucher specimens (with an identification code as stated in parentheses above) deposited in the botanical herbarium at the Faculty of Agricultural Sciences, University of Guilan, Iran. The leaves collected were clean, dried oven at $45^{\circ} \mathrm{C}$, and milled. The milled leaves had been suspended in the ethanol $70 \%$ and water for extract preparation.

For aqueous extract, $1 \mathrm{~g}$ of plant tissue was put in a $90^{\circ} \mathrm{C}$ container with $10 \mathrm{ml}$ tap water. After shaking for $60 \mathrm{~min}$, the filter paper Whatman No. 2 was used to separate the extract, which was then diluted from $10^{-1}$ to $10^{-7}$. For ethanolic extraction, each plant was dried at room temperature and $1 \mathrm{~g}$ of each powdered plant material was soaked in $15 \mathrm{ml}$ of ethanol $70 \%$ for $12 \mathrm{~h}$ with continuous vigorous shaking at $30 \mathrm{~min}$ intervals. The suspension was allowed to settle, and the liquid phase passed through a filter paper Whatman No. 1 and dehydrated at $80^{\circ} \mathrm{C}$ in a rotary evaporator. Stock final solutions of crude extracts were prepared by mixing the appropriate amount of dimethyl sulfoxide (DMSO) thoroughly to 
obtain a final concentration. The final solutions were filtered through a DMSO-compatible sterilized 0.22 filter and stored at $4{ }^{\circ} \mathrm{C}$ until applied for in vitro assay of antimicrobial activity $[28,29,30]$.

For essential oil extraction, fresh aerial sections of Eryngium caucasicum Trautv were air-dried for a week at $25^{\circ} \mathrm{C}$ in the shade, cut into small pieces and milled to a homogeneous powder. The powder was immediately put in a $2 \mathrm{~L}$ flask containing an appropriate amount of distilled water. Then, $300 \mathrm{~g}$ aerial sections powder of Eryngium caucasicum Trautv was subjected to hydrodistillation for $3 \mathrm{~h}$ with $1 \mathrm{~L}$ distilled water using a Clevenger-type apparatus according to the European Pharmacopoeia. The extracted oil was collected and dried with anhydrous sodium sulphate and stored at $4{ }^{\circ} \mathrm{C}$ prior to analysis [31].

\section{Antimicrobial activity of plant products}

Agar well diffusion and microdilution procedures have been used to determine the antimicrobial efficacy of extracts and essential oil (ethanolic and aqueous extracts of Eryngium caucasicum Trautv = EtEc and AqEc; essential oil, ethanolic and aqueous extracts of Froriepia subpinnata= EoFs, EtFs and AqFs). The agar well diffusion method was carried out according to the National Clinical Laboratory Quality Committee [32]. After preparing the Mueller Hinton Agar (MHA) medium, $6 \mathrm{~mm}$ diameter wells were made on this medium using a cork borer. Then, inoculum containing $1.5 \times 10^{8} \mathrm{CFU} / \mathrm{ml}(0.5 \mathrm{McF}$ arland turbidity standard) of each bacterial culture to be tested has been spread with a sterile swab moistened with bacterial suspension on MHA plate. Fifty microliter of each concentration of the extracts $\left(10^{-1}\right.$ to $\left.10^{-7}\right)$ was added into the wells that were punched on the plates and allowed to diffuse at room temperature for $2 \mathrm{~h}$. Subsequently, the plates were incubated at $37^{\circ} \mathrm{C}$ for $24 \mathrm{~h}$. The diameters of the growth inhibition zones were determined after incubation.

Minimum inhibitory concentration (MIC) and the minimum bactericidal concentration (MBC) of crude plant extracts and essential oil were determined using broth microdilution method. Seven dilutions of the $10^{-1}$ to $10^{-7}$ samples were evaluated. Test isolate inoculums prepared from fresh overnight cultures were adjusted to $0.5 \mathrm{McF}$ arland standards, which is equal to $1.5 \times 10^{8} \mathrm{CFU} / \mathrm{ml}$. The $50 \mu \mathrm{l}$ sample was poured into each 96-well microplate well which contained $50 \mu \mathrm{l}$ of Mueller Hinton Broth. Then $50 \mu \mathrm{l}$ of test strain suspensions were added. The highest dilution of samples with no visible growth or turbidity and no growth after incubation at $37^{\circ} \mathrm{C}$ for $24 \mathrm{~h}$ was considered as MIC and MBC, respectively [33].

\section{Phytochemicl assay}

Reversed-phase High- performance liquid chromatography (RP-HPLC) with UV detection was used to analyze polyphenols including chlorogenic acid and also flavonoids including quercetin and catechin. One gram of dried and powdered leaves was extracted from each sample with $6 \mathrm{ml}$ of methanol-and acetic acid extraction solvent $(85: 15, \mathrm{v} / \mathrm{v})$. The collected extract was filtered $(0.45 \mu \mathrm{m})$ and the filtrate was concentrated and dried using a rotary vacuum evaporator. The dried extract was being dissolved in the mobile phase. Fifty microliters of sample was injected in HPLC (Waters, 1525, Milford, USA) equipped with a UV-Visible detector (Waters Dual $\lambda$ Absorbance 2487), C18 column: Waters Symmetry C18 $5 \mu \mathrm{m} 5$ $\times 150 \mathrm{~mm}$ (Waters, Dublin, Ireland), at 280 and $320 \mathrm{~nm}$. HPLC chromatograms have been detected using 
a photo diode array Ultra-Violet (UV) detector at two different wavelengths (280 and $320 \mathrm{~nm}$ ) according to the maximum absorption of the analyzed compounds. The calculation of the integrated peak area was used to quantify chlorogenic acid and flavonoids in the sample extracts, and the contents were determined using the calibration curve by plotting peak area against concentration of the respective standard sample [34].

The Gas chromatography-mass spectrometry (GC-MS) analysis was carried out using an Agilent 6850 gas chromatograph equipped with an Agilent 7890A mass spectrometer for determining of EoFs ingredients. The ingredients were separated by HP-5MS UI capillary column including $5 \%$ phenyl polysiloxane as stationary phase, $30 \mathrm{~m}$ length, $0.25 \mathrm{~mm}$ internal diameter and $0.25 \mu \mathrm{m}$ thickness. The temperature of the injector was $300^{\circ} \mathrm{C}$ and $1.0 \mu \mathrm{l}$ of the sample was injected in split mode. Helium was applied as a carrier gas with the split ratio 1:50 and flow-rate of $1 \mathrm{ml} / \mathrm{min}$. The column temperature program was $60^{\circ} \mathrm{C}$ during $5 \mathrm{~min}$, with $3{ }^{\circ} \mathrm{C} / \mathrm{min}$ increases to $180^{\circ} \mathrm{C}$, then $20^{\circ} \mathrm{C} / \mathrm{min}$ increases to $280^{\circ} \mathrm{C}$, which was held for $10 \mathrm{~min}$. The determination of mass spectra was performed in electron ionization (EI) mode, with ionization energy of $70 \mathrm{eV}$, while the scanned mass range was $3-500 \mathrm{~m} / \mathrm{z}$. Component identification was based on a comparison of their mass spectrums with those of the NIST mass spectral library $[35,36]$.

\section{Statistical analysis}

All measurements have been replicated three times, and data was reported as mean $\pm S D$. The results were statistically evaluated using a one-way variance analysis (ANOVA) and the variations between the means were calculated using the multiple range tests of Duncan at $P \leq 0.01$.

\section{Results}

\section{Antibiotic susceptibility}

Of the 138 urine samples included in this research, 112 have been contaminated with bacteria from which 91 have been positive for $E$. coli. E coli isolates identified by a distinctive greenish metallic sheen on EMB, pink colored colonies on the MacConkey agar and beta-hemolysis on blood agar. The nitrate reduction, indole, methyl-red, catalase, lysine decarboxylase, $\mathrm{CO}_{2}, \mathrm{ONPG}$ and glucose, lactose, mannitol, mlatose fermentation tests were positive for all $91 \mathrm{E}$. coli isolates, and the citrate, oxidase, urease, Voges Proskuaer, and $\mathrm{H}_{2} \mathrm{~S}$ production tests were negative for all strains. These findings indicate that these isolates belong to $E$. coli. Since the susceptibility pattern of all isolates differed from the 20 antibiotics used, all isolates were distinct. The antibiotic susceptibility profile of all isolates to the 20 antibiotics used is shown in Fig. 1. Considering that all isolates used were resistant to at least three antibiotics from three different families, all isolates could be introduced as MDR. Generally, carbapenems, including meropenem and emipenem, were the most effective class of antibiotics affecting $E$. coli isolates by affecting $91.90 \% \pm 1.89$ and $65.34 \% \pm 1.39$ of bacteria, respectively, and penicillins, with the exception of pipiracylin, which was the most effective antibiotic after meropenem, other penicillins were classified as the least potency antibiotics. 


\section{Antimicrobial activity of plant products}

The antimicrobial activity of herbal products with different concentration $(11.11,1.11,0.11$ and $0.011 \mathrm{mg} / \mathrm{ml}$ ) against MDR E. coli isolates used in this research is evaluated using agar well diffusion and microdilution methods. Agar well diffusion method has been used for the initial detection of antimicrobial activity of products on certain isolates. After confirming the antimicrobial activity of certain products using this method, the microdilution method was used to assess the effective quantity of products as MIC and MBC on sensitive isolates.

The results of the diffusion agar gel method given in Table 1 include the mean of growth inhibition zone diameter with standard deviation of the lowest product concentration affecting the isolates. In other words, those values for the products listed in Table 1 are in fact the product's lowest concentrations affecting the particular isolate. Many products don't have any effect on certain isolates based on this method. Some products also have an effect on one isolate, but others do not. The effect of the product on different isolates was similar in some cases, and different under other instances. The activity of different products on one or more isolates was the same for some isolates and different for other isolates. As shown in Fig. 2, EoFs had the greatest potency with a growth inhibition diameter of $23 \pm$ $0.38 \mathrm{~mm}$, although other products also produced a growth inhibition zone with a diameter approximately close to EoFs. The reason for the introduction of EoFs as the most effective product compared to other products is that it has had an inhibitory and killing effect on more isolates and, in most cases, has shown its activity at lower concentrations. In most antibiotic-susceptible isolates, the growth inhibition zone diameter begins at a minimum of 15-16 mm, according to the CLSI table. In general, the diameter of the growth inhibition zone on antibiotic-susceptible isolates was higher as compared to plant products. 
Table 1

Antimicrobial activity of the plant extracts evaluated by agar well diffusion method.

Mean diameter of growth inhibition zone \pm standard deviation $(\mathrm{mm})$

\begin{tabular}{|c|c|c|c|c|c|}
\hline E. coli isolates & EoFs & EtFs & AqFs & EtEc & $\mathrm{AqEc}$ \\
\hline E1 & - & - & - & - & - \\
\hline E2 & $14 \pm 0.12^{b}$ & - & - & $19 \pm 0.21^{a}$ & - \\
\hline E3 & $18 \pm 0.32^{\mathrm{a}}$ & - & - & - & - \\
\hline E4 & $13 \pm 0.72^{b}$ & $20 \pm 0.32^{a}$ & $19 \pm 0.32^{a}$ & $14 \pm 0.26^{b}$ & - \\
\hline E5 & - & - & - & - & - \\
\hline E6 & - & - & - & - & - \\
\hline E7 & - & - & - & - & - \\
\hline E8 & $22 \pm 0.78^{a}$ & $20 \pm 0.34^{a}$ & $17 \pm 041^{a}$ & - & - \\
\hline E9 & $20 \pm 043^{a}$ & - & - & - & - \\
\hline E10 & $22 \pm 0.26^{\mathrm{a}}$ & $12 \pm 0.13^{c}$ & $18 \pm 0.84^{a}$ & - & $14 \pm 0.86^{b}$ \\
\hline E11 & - & - & - & - & - \\
\hline E12 & $15 \pm 0.12^{b}$ & $13 \pm 0.21^{b}$ & $17 \pm 0.88^{a}$ & $19 \pm 0.22^{a}$ & $19 \pm 0.48^{a}$ \\
\hline E13 & - & $18 \pm 0.56^{a}$ & $17 \pm 0.64^{a}$ & - & - \\
\hline E14 & - & - & - & - & - \\
\hline E15 & - & - & - & - & - \\
\hline E16 & - & - & - & - & - \\
\hline E17 & $16 \pm 0.45^{b}$ & $19 \pm 0.32^{\mathrm{a}}$ & $19 \pm 0.48^{\mathrm{a}}$ & $20 \pm 0.65^{a}$ & - \\
\hline E18 & $23 \pm 0.38^{a}$ & $18 \pm 0.46^{b}$ & - & - & - \\
\hline E19 & $20 \pm 0.73^{a}$ & - & - & - & - \\
\hline E20 & $15 \pm 0.21^{b}$ & $17 \pm 0.28^{a}$ & $17 \pm 0.46^{\mathrm{a}}$ & $13 \pm 0.84^{b}$ & - \\
\hline E21 & $18 \pm 0.36^{a}$ & - & - & - & - \\
\hline
\end{tabular}

EoFs: essential oil of Eryngium caucasicum Trautv; EtFs: ethanolic extract of Froriepia subpinnata; AqFs: aqueous extract of Froriepia subpinnata; EtEc: ethanolic extract of Eryngium caucasicum Trautv; AqEc: aqueous extract of Eryngium caucasicum Trautv). The letters a, b, c and d indicate concentrations of $11.11,1.11,0.11$ and $0.011 \mathrm{mg} / \mathrm{ml}$, respectively. 


\section{Mean diameter of growth inhibition zone \pm standard deviation $(\mathrm{mm})$}

\begin{tabular}{|c|c|c|c|c|c|}
\hline E22 & - & - & - & - & - \\
\hline E23 & - & - & - & - & - \\
\hline E24 & $21 \pm 0.48^{a}$ & $15 \pm 0.37^{b}$ & $14 \pm 0.46^{b}$ & $20 \pm 0.24^{a}$ & - \\
\hline E25 & $22 \pm 0.33^{a}$ & $9 \pm 0.24^{c}$ & - & $18 \pm 0.44^{a}$ & $14 \pm 0.24^{b}$ \\
\hline E26 & - & - & - & - & - \\
\hline E27 & - & - & - & - & - \\
\hline E28 & $14 \pm 0.44^{b}$ & $18 \pm 0.26^{a}$ & $17 \pm 0.68^{a}$ & $20 \pm 0.38^{a}$ & - \\
\hline E29 & $22 \pm 0.44^{\mathrm{a}}$ & $18 \pm 0.36^{a}$ & $17 \pm 0.26^{a}$ & - & - \\
\hline E30 & $16 \pm 0.48^{a}$ & $14 \pm 0.64^{b}$ & $13 \pm 0.46^{c}$ & $15 \pm 0.64^{b}$ & $17 \pm 0.46^{a}$ \\
\hline E31 & $16 \pm 0.13^{b}$ & $18 \pm 0.65^{a}$ & - & $16 \pm 0.45^{b}$ & - \\
\hline E32 & $8 \pm 0.12^{c}$ & $13 \pm 0.26^{b}$ & $18 \pm 0.35^{a}$ & $12 \pm 0.48^{c}$ & $14 \pm 0.28^{b}$ \\
\hline E33 & $13 \pm 0.24^{b}$ & $18 \pm 0.64^{a}$ & $17 \pm 0.46^{a}$ & $12 \pm 0.75^{c}$ & - \\
\hline E34 & - & - & - & - & - \\
\hline E35 & $22 \pm 0.14^{\mathrm{a}}$ & - & - & - & - \\
\hline E36 & $13 \pm 0.34^{b}$ & - & - & - & - \\
\hline E37 & $17 \pm 0.86^{a}$ & - & - & - & - \\
\hline E38 & - & - & - & - & - \\
\hline E39 & - & - & - & - & - \\
\hline E40 & - & - & - & - & - \\
\hline E41 & - & - & - & - & - \\
\hline E42 & $20 \pm 0.64^{\mathrm{a}}$ & $18 \pm 0.76^{a}$ & $17 \pm 0.35^{a}$ & - & - \\
\hline E43 & - & - & - & - & - \\
\hline E44 & - & - & - & - & - \\
\hline
\end{tabular}

EoFs: essential oil of Eryngium caucasicum Trautv; EtFs: ethanolic extract of Froriepia subpinnata; AqFs: aqueous extract of Froriepia subpinnata; EtEc: ethanolic extract of Eryngium caucasicum Trautv; AqEc: aqueous extract of Eryngium caucasicum Trautv). The letters a, b, c and d indicate concentrations of $11.11,1.11,0.11$ and $0.011 \mathrm{mg} / \mathrm{ml}$, respectively. 


\section{Mean diameter of growth inhibition zone \pm standard deviation (mm)}

\section{E45}

E46

$13 \pm 0.45^{b}$

$19 \pm 0.54^{\mathrm{a}}$

$17 \pm 0.64^{\mathrm{a}}$

$20 \pm 0.24^{a}$

E47

$20 \pm 0.26^{\mathrm{a}}$

$14 \pm 0.45^{b}$

$19 \pm 0.67^{a}$

$18 \pm 0.26^{a}$

E48

E49

$15 \pm 0.44^{b}$

$20 \pm 0.24^{a}$

$20 \pm 0.32^{\mathrm{a}}$

$21 \pm 0.26^{a}$

E50

$12 \pm 0.64^{c}$

$15 \pm 0.24^{b}$

$18 \pm 0.64^{a}$

$15 \pm 0.32^{b}$

$18 \pm 0.64^{a}$

E51

E52

E53

$12 \pm 0.24^{c}$

$18 \pm 0.36^{b}$

$13 \pm 0.64^{b}$

$16 \pm 0.75^{b}$

$18 \pm 0.24^{\mathrm{a}}$

E54

E55

$22 \pm 0.36^{\mathrm{a}}$

$20 \pm 0.32^{\mathrm{a}}$

$20 \pm 0.46^{\mathrm{a}}$

E56

E57

$19 \pm 0.46^{a}$

E58

$15 \pm 0.22^{b}$

$18 \pm 0.64^{a}$

$18 \pm 0.84^{\mathrm{a}}$

$18 \pm 0.33^{a}$

E59

$12 \pm 0.22^{c}$

$13 \pm 0.48^{b}$

$11 \pm 0.76^{c}$

$16 \pm 0.24^{\mathrm{b}}$

$18 \pm 0.64^{\mathrm{a}}$

E60

$15 \pm 0.36^{b}$

$14 \pm 0.48^{b}$

$13 \pm 0.76^{b}$

$19 \pm 0.64$

$15 \pm 0.22^{b}$

E61

E62

E63

$14 \pm 0.46^{b}$

$18 \pm 0.66^{a}$

$-$

$18 \pm 0.55^{a}$

$22 \pm 0.76^{a} \quad 18 \pm 0.43^{a} \quad 17 \pm 0.36^{a}$

E64

$13 \pm 0.22^{b}$
-
$20 \pm 0.22^{a}$

E67

EoFs: essential oil of Eryngium caucasicum Trautv; EtFs: ethanolic extract of Froriepia subpinnata; AqFs: aqueous extract of Froriepia subpinnata; EtEc: ethanolic extract of Eryngium caucasicum Trautv; AqEc: aqueous extract of Eryngium caucasicum Trautv). The letters a, b, c and d indicate concentrations of $11.11,1.11,0.11$ and $0.011 \mathrm{mg} / \mathrm{ml}$, respectively. 


\section{Mean diameter of growth inhibition zone \pm standard deviation $(\mathrm{mm})$}

E68

E69

E70

E71

E72

E73

E74

E75

E76

E77

E78

$14 \pm 0.76^{b}$

$15 \pm 0.34^{\mathrm{b}}$

$20 \pm 0.46^{\mathrm{a}}$

$15 \pm 0.54^{\mathrm{b}}$

E79

E80

$12 \pm 0.44^{c}$

$15 \pm 0.36^{b}$

$18 \pm 0.74^{\mathrm{a}}$

$14 \pm 0.66^{b}$

E81

E82

E83

E84

$12 \pm 0.26^{c}$

$15 \pm 0.87^{b}$

$20 \pm 0.67^{a}$

$16 \pm 0.55^{\mathrm{b}}$

E85

E86

E87

E88

E89

E90

$14 \pm 0.44^{\mathrm{b}}$
$23 \pm 0.87^{\mathrm{a}}$
-

$18 \pm 0.64^{\mathrm{a}} \quad 17 \pm 0.87^{\mathrm{a}} \quad 17 \pm 0.66^{\mathrm{a}}$

$20 \pm 0.36^{a}$

$18 \pm 0.56^{\mathrm{a}}$

$15 \pm 0.26^{b}$
$6 \pm 0.12^{d}$

$15 \pm 0.74^{b}$

$14 \pm 0.21^{b}$

$17 \pm 0.64^{\mathrm{a}}$

$11 \pm 0.46^{\mathrm{c}}$

$12 \pm 0.55^{\mathrm{c}}$

$7 \pm 0.022^{d}$

$18 \pm 0.87^{a}$

$15 \pm 0.87^{b}$

EoFs: essential oil of Eryngium caucasicum Trautv; EtFs: ethanolic extract of Froriepia subpinnata; AqFs: aqueous extract of Froriepia subpinnata; EtEc: ethanolic extract of Eryngium caucasicum Trautv; AqEc: aqueous extract of Eryngium caucasicum Trautv). The letters a, b, c and d indicate concentrations of $11.11,1.11,0.11$ and $0.011 \mathrm{mg} / \mathrm{ml}$, respectively. 


\section{Mean diameter of growth inhibition zone \pm standard deviation $(\mathrm{mm})$}

E91

$10 \pm 0.44^{c}$

$12 \pm 0.76^{c}$

$15 \pm 0.24^{b}$

$14 \pm 0.54^{b}$

$14 \pm 0.76^{\mathrm{b}}$

EoFs: essential oil of Eryngium caucasicum Trautv; EtFs: ethanolic extract of Froriepia subpinnata; AqFs: aqueous extract of Froriepia subpinnata; EtEc: ethanolic extract of Eryngium caucasicum Trautv; AqEc: aqueous extract of Eryngium caucasicum Trautv). The letters a, b, c and d indicate concentrations of $11.11,1.11,0.11$ and $0.011 \mathrm{mg} / \mathrm{ml}$, respectively.

To evaluate the effective quantity of products as MIC and MBC, microdilution method was applied in isolates that were susceptible to the plant products based on the gel diffusion method. As shown in Fig. 2, EoFs and AqEc had the highest and lowest inhibitory and killing effects on all isolates, respectively. Ethanolic extracts were more effective than aqueous extracts. The antimicrobial effects of Froriepia subpinnata products have been slightly higher than those of Eryngium caucasicum Trautv. No inhibitory or killing effects of herbal products were observed at concentrations below $0.011 \mathrm{mg} / \mathrm{ml}$. Some of the products used did not affect the isolates. Generally, the herbal products inhibitory impacts on the total

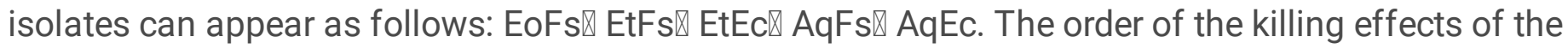
products can also be shown on the whole isolates as follows: EoFs $\mathbb{E t E c} \rrbracket \mathrm{EtFs} \rrbracket \mathrm{AqFs} \bigotimes \mathrm{AqEc}$.

MIC values of the tested plant materials generally varied within the range from 0.011 to $11.11 \mathrm{mg} / \mathrm{ml}$ according to obtained data, and in MIC less than $0.011 \mathrm{mg} / \mathrm{ml}$, none of the products has any effect on any of the isolates. As can be seen from Table 2, Froriepia subpinnata and Eryngium caucasicum Trautv products had no effect on 38 and 53 isolates, respectively. No antimicrobial activity was observed from Froriepia subpinnata products including EoFs, EtFs, and AqFs against 41, 51 and 56 isolates, respectively. Contrary to the extracts, the antimicrobial effects of EoFs have been identified on 15 isolates. The extracts were effective on only one isolate, but the EoFs did not have any effect. In most cases, EoFs MIC was lower than extracts at a similar concentration. The efficacy of EtFs and AqFs was the same in cases in which the extracts had an effect on the isolates, with the exception of 16 isolates. 
Table 2

MIC and MBC value $(\mathrm{mg} / \mathrm{ml})$ of plant samples based on microdilution method on $91 \mathrm{MDR} E$. coli isolates.

\begin{tabular}{|c|c|c|c|c|c|c|c|c|c|c|}
\hline & MIC & & & $\mathrm{MBC}$ & & & MIC & & $\mathrm{MBC}$ & \\
\hline $\begin{array}{l}\text { E. coli } \\
\text { isolates }\end{array}$ & EoFs & EtFs & AqFs & EoFs & EtFs & AqFs EtEc & & $\mathrm{AqEc}$ & EtEc & $\mathrm{AqEc}$ \\
\hline E1 & - & - & - & - & - & - & - & - & - & - \\
\hline E2 & 1.11 & - & - & 11.11 & - & - & - & - & - & - \\
\hline E3 & 11.11 & - & - & - & - & - & 11.11 & - & - & - \\
\hline E4 & 1.11 & 11.11 & 11.11 & 1.11 & - & - & - & - & - & - \\
\hline E5 & - & - & - & - & - & - & - & - & - & - \\
\hline E6 & - & - & - & - & - & - & - & - & - & - \\
\hline E7 & - & - & - & - & - & - & - & - & - & - \\
\hline E8 & 11.11 & 11.11 & 11.11 & - & - & - & 1.11 & 11.11 & 11.11 & 11.11 \\
\hline E9 & 11.11 & - & - & - & - & - & - & - & - & - \\
\hline E10 & 11.11 & 0.11 & 11.11 & - & 1.11 & - & 1.11 & 1.11 & 1.11 & 11.11 \\
\hline E11 & - & - & - & - & - & - & - & - & - & - \\
\hline E12 & 1.11 & 1.11 & 11.11 & 11.11 & 11.11 & - & - & - & - & - \\
\hline E13 & - & 11.11 & 11.11 & - & - & - & 11.11 & 11.11 & - & - \\
\hline E14 & - & - & - & - & - & - & 11.11 & 11.11 & 11.11 & - \\
\hline E15 & - & - & - & - & - & - & - & - & - & - \\
\hline E16 & - & - & - & - & - & - & 11.11 & 11.11 & - & - \\
\hline E17 & 1.11 & 11.11 & 11.11 & 11.11 & - & - & - & - & - & - \\
\hline E18 & 11.11 & 11.11 & - & - & - & - & - & - & - & - \\
\hline E19 & 11.11 & - & - & - & - & - & - & - & - & - \\
\hline E20 & 1.11 & 11.11 & 11.11 & 1.11 & - & - & - & - & - & - \\
\hline E21 & 11.11 & - & - & - & - & - & 1.11 & - & 1.11 & - \\
\hline E22 & - & - & - & - & - & - & 11.11 & - & - & - \\
\hline E23 & - & - & - & - & - & - & - & - & - & - \\
\hline E24 & 11.11 & 1.11 & 1.11 & 11.11 & - & - & - & - & - & - \\
\hline E25 & 11.11 & 0.11 & - & 11.11 & 1.11 & 1.11 & - & - & - & - \\
\hline
\end{tabular}




\begin{tabular}{|c|c|c|c|c|c|c|c|c|c|c|}
\hline & MIC & & & $\mathrm{MBC}$ & & & MIC & & $\mathrm{MBC}$ & \\
\hline E26 & - & - & - & - & - & - & 1.11 & 11.11 & 11.11 & 11.11 \\
\hline E27 & - & - & - & - & - & - & 0.011 & 1.11 & 0.11 & 11.11 \\
\hline E28 & 1.11 & 11.11 & 11.11 & 11.11 & - & - & - & - & - & - \\
\hline E29 & 11.11 & 11.11 & 11.11 & - & - & - & - & - & - & - \\
\hline E30 & 1.11 & 1.11 & 1.11 & 1.11 & 11.11 & 11.11 & - & - & - & - \\
\hline E31 & 1.11 & 11.11 & - & 1.11 & - & - & - & - & - & - \\
\hline E32 & 0.011 & 1.11 & 11.11 & 0.11 & 1.11 & 1.11 & 0.11 & 1.11 & 1.11 & 1.11 \\
\hline E33 & 0.11 & 11.11 & 11.11 & 0.11 & - & - & 1.11 & 1.11 & 11.11 & 11.11 \\
\hline E34 & - & - & - & - & - & - & 11.11 & 11.11 & - & - \\
\hline E35 & 11.11 & - & - & - & - & - & - & - & - & - \\
\hline E36 & 0.11 & - & - & - & - & - & - & - & - & - \\
\hline E37 & 11.11 & - & - & - & - & - & - & - & - & - \\
\hline E38 & - & - & - & - & - & - & 11.11 & 11.11 & - & - \\
\hline E39 & - & - & - & - & - & - & - & - & - & - \\
\hline E40 & - & - & - & - & - & - & - & - & - & - \\
\hline E41 & - & - & - & - & - & - & 11.11 & 11.11 & - & - \\
\hline E42 & 11.11 & 11.11 & 11.11 & - & - & - & 1.11 & 11.11 & 1.11 & - \\
\hline E43 & - & - & - & - & - & - & - & - & - & - \\
\hline E44 & - & - & - & - & - & - & - & - & - & - \\
\hline E45 & - & - & - & - & - & - & - & - & - & - \\
\hline E46 & 1.11 & 11.11 & 11.11 & 11.11 & - & - & - & - & - & - \\
\hline E47 & 11.11 & 1.11 & 11.11 & - & 11.11 & - & - & - & - & - \\
\hline E48 & - & - & - & - & - & - & - & - & - & - \\
\hline E49 & 1.11 & 11.11 & 11.11 & 11.11 & - & - & 1.11 & 11.11 & 11.11 & 11.11 \\
\hline E50 & 0.11 & 1.11 & 11.11 & 1.11 & 11.11 & 11.11 & - & - & - & - \\
\hline E51 & - & - & - & - & - & - & 11.11 & - & - & - \\
\hline E52 & - & - & - & - & - & - & 1.11 & 11.11 & 1.11 & - \\
\hline E53 & 0.11 & 11.11 & 1.11 & 1.11 & 11.11 & 11.11 & 0.011 & 0.11 & 0.011 & 1.11 \\
\hline
\end{tabular}




\begin{tabular}{|c|c|c|c|c|c|c|c|c|c|c|}
\hline & MIC & & & MBC & & & MIC & & MBC & \\
\hline E54 & - & - & - & - & - & - & - & - & - & - \\
\hline E55 & 11.11 & 11.11 & 11.11 & - & - & - & - & - & - & - \\
\hline E56 & - & - & - & - & - & - & - & - & - & - \\
\hline E57 & 11.11 & - & - & - & - & - & 1.11 & 11.11 & 1.11 & - \\
\hline E58 & 1.11 & 11.11 & 11.11 & 11.11 & - & - & - & - & - & - \\
\hline E59 & 0.11 & 1.11 & 0.11 & 1.11 & 11.11 & 1.11 & 0.11 & 1.11 & 0.11 & 1.11 \\
\hline E60 & 1.11 & 1.11 & 1.11 & 11.11 & 3 & 11.11 & - & - & - & - \\
\hline E61 & - & - & - & - & - & - & - & - & - & - \\
\hline E62 & 1.11 & 11.11 & - & 11.11 & - & - & - & - & - & - \\
\hline E63 & 11.11 & 11.11 & 11.11 & - & - & - & - & - & - & - \\
\hline E64 & 0.11 & 1.11 & 1.11 & 1.11 & 11.11 & 11.11 & - & - & - & - \\
\hline E65 & - & - & - & - & - & - & - & - & - & - \\
\hline E66 & 11.11 & - & - & - & - & - & 1.11 & 11.11 & 11.11 & - \\
\hline E67 & - & - & - & - & - & - & 11.11 & - & - & - \\
\hline E68 & - & - & - & - & - & - & 1.11 & 11.11 & 11.11 & - \\
\hline E69 & 11.11 & - & - & 11.11 & - & - & 1.11 & 1.11 & 11.11 & 11.11 \\
\hline E70 & - & - & - & - & - & - & - & - & - & - \\
\hline E71 & 0.11 & 0.11 & 1.11 & 1.11 & 1.11 & 1.11 & - & - & - & - \\
\hline E72 & 11.11 & 11.11 & - & - & - & & 11.11 & 11.11 & - & - \\
\hline E73 & - & - & - & - & - & - & - & - & - & - \\
\hline E74 & - & - & - & - & - & - & - & - & - & - \\
\hline E75 & 0.11 & 1.11 & 11.11 & 1.11 & 11.11 & - & - & - & - & - \\
\hline E76 & - & - & - & - & - & - & - & - & - & - \\
\hline E77 & - & - & - & - & - & - & 1.11 & - & 11.11 & - \\
\hline E78 & 1.11 & 1.11 & 11.11 & 1.11 & 11.11 & 11.11 & - & - & - & - \\
\hline E79 & - & - & - & - & - & - & 11.11 & 11.11 & - & - \\
\hline E80 & 0.11 & 1.11 & 11.11 & 1.11 & 11.11 & - & 1.11 & 11.11 & 1.11 & - \\
\hline E81 & - & - & - & - & - & - & 11.11 & 11.11 & - & - \\
\hline
\end{tabular}




\begin{tabular}{|lllllllllll|}
\hline & MIC & \multicolumn{3}{c}{ MBC } & \multicolumn{3}{c|}{ MIC } & \multicolumn{3}{c|}{ MBC } \\
\hline E82 & - & - & - & - & - & - & 1.11 & 1.11 & 11.11 & 11.11 \\
\hline E83 & - & - & - & - & - & - & - & - & - & - \\
E84 & 0.11 & 1.11 & 11.11 & 1.11 & 11.11 & 11.11 & - & - & - & - \\
\hline E85 & - & - & - & - & - & - & - & - & - & - \\
\hline E86 & 1.11 & 11.11 & 11.11 & 11.11 & - & - & - & - & - & - \\
E87 & 11.11 & 11.11 & - & 11.11 & - & - & - & - & - & - \\
E88 & - & - & - & - & - & - & - & - & - & - \\
\hline E89 & 1.11 & 1.11 & 1.11 & 11.11 & 11.11 & 11.11 & 1.11 & 11.11 & 11.11 & - \\
\hline E90 & 0.011 & 0.11 & 0.11 & 0.011 & 1.11 & 1.11 & 1.11 & 11.11 & 1.11 & 11.11 \\
\hline E91 & 0.11 & 0.11 & 1.11 & 1.11 & 1.11 & 11.11 & 11.11 & - & - & - \\
\hline
\end{tabular}

In Eryngium caucasicum Trautv, the effect of EtEc on 9 isolates was greater than that of the AqEb extract in cases where the extracts had an effect on the isolates, with the exception of 13 cases in which the effect of the extracts was equal at similar concentrations. The antimicrobial activity of EtEc and AqEc were detected on 38 and 29 isolates, respectively.

\section{Plants products in comparison with antibiotics}

A comparison of the inhibitory activity of 20 antibiotics used with the different concentrations of herbal products, including $11.11,1.11,0.11$ and $0.011 \mathrm{mg} / \mathrm{ml}$ on a total of $91 \mathrm{MDR}$ E. coli isolates is shown in Fig. 2. The most effective inhibitor, EoFs, except for the meropenem, imipenem, piperacillin, chloramphenicol, cephalothin and cefepime antibiotics, showed more inhibitory activity than the other 14 antibiotics $(P<0 / 05)$.

EtFs was the second product with the highest antimicrobial activity, displaying greater inhibitory potency than 10 antibiotics, except for piperacillin, fosfomycin, ciprofloxacin, streptomycin, tetracycline, chloramphenicol, cephalothin, cefotaxime, cefixime, ceftazidime, cefepime, imipenem and meropenem ( $P$ $<0 / 05)$. However, the lowest inhibitory activity is related to AqEc, which has more inhibitory activity than 7 antibiotics except piperacillin, fosfomycin, ciprofloxacin, chloramphenicol, cephalothin, cefotaxime, ceftazidim, cefepime, imipenem and meropenem $(P<0 / 05)$.

\section{Phytochemical assay}

The HPLC analysis was applied to identify and quantify catechin and quercetin flavonoids as well as chlorogenic acid polyphenol. The chromatograms obtained from the flavonoid and polyphenol standard 
samples and the samples related to the ethanolic and aqueous extracts studied are shown in Fig. 3, Fig. 4 and Fig. 5. The highest level of catechin and quercetin was detected in AqEc among the extracts.

In Froriepia subpinnata, more catechins were found in the ethanolic extract than the aqueous extract, but quercetin was higher in the aqueous extract than the ethanolic extract, whereas in Eryngium caucasicum Trautv both flavonoids were higher in the aqueous extract than the ethanolic extract. Catechin was found in extracts containing EtFs, EtEc and AqFs in the amounts $20.44 \mathrm{~g} / 100 \mathrm{~g} \pm 1.87 \mathrm{DW}, 11.36 \pm 1.22 \mathrm{~g} / 100 \mathrm{~g}$ DW and $45.78 \pm 1.84 \mathrm{~g} / 100 \mathrm{~g} \mathrm{DW}$, respectively, with the exception of the AqFs. In comparison to chlorogenic acid, which was not detectable in any of the products, quercetin was detectable in all products containing EtFs, AqFs, EtEc and AqEc with $0.50 \pm 0.01 \mathrm{~g} / 100 \mathrm{~g} \mathrm{DW}, 44.03 \pm 1.87 \mathrm{~g} / 100 \mathrm{~g} \mathrm{DW}$, $18.50 \pm 0.14 \mathrm{~g} / 100 \mathrm{~g} \mathrm{DW}$ and $69.74 \pm 2.24 \mathrm{~g} / 100 \mathrm{~g} \mathrm{DW}$ values, respectively.

GC-mass chromatogram and EoFs components are shown in Fig. 6 and Table 3, respectively. The number and type of phytochemical components as shown by the peaks were characterized and described by comparing the constituent mass spectra to the NIST library. Phytochemical assay of EoFs using GC-mass analysis detected 24 peaks correspond to the 10 types of compounds. These compounds include monoterpenoid, alcohol, alkane, cycloalkane, cycloalkene, ketone, aldehyde, phenol derivative, furan derivatives and acid, which comprise $2.03 \%, 9.73 \%, 7.03 \%, 22.24 \%, 24.04 \%, 5.51 \%, 7.46 \%, 1.19 \%, 3.33 \%$ and $1.77 \%$ of EoFs, respectively. As is evident from the data, cycloalkene and cycloalkane are the most abundant EoFs components. 
Table 3

The EoFs components deteced by GC-mass analysis.

\begin{tabular}{|c|c|c|c|c|}
\hline $\begin{array}{l}\text { Peak } \\
\text { No. }\end{array}$ & Compounds & RT & $\begin{array}{l}\text { Area } \\
(\%)\end{array}$ & $\begin{array}{l}\text { Probability } \\
(\%)\end{array}$ \\
\hline 1 & Benzenemethanol, 4-(1-methylethyl) & 13.48 & 5.78 & 91 \\
\hline 2 & Benzenemethanol, .alpha.,.alpha. -trimethyl & 15.53 & 1.43 & 91 \\
\hline 3 & $\begin{array}{l}\text { (-)-(1S,5R)-endo-2(R),8,8-Trimethy lbicyclo[3.2.1]octan-3- } \\
\text { one }\end{array}$ & 16.83 & 14.33 & 64 \\
\hline 4 & 3-Furaldehyde & 18.07 & 7.46 & 30 \\
\hline 5 & Linalool & 18.83 & 2.03 & 42 \\
\hline 6 & Phenol, 5-methyl-2-(1-methylethyl) & 19.15 & 1.19 & 95 \\
\hline 7 & 2,5-Dimethyl-4-vinyl-hex-5-en-2,3- diol & 19.61 & 0.88 & 28 \\
\hline 8 & 6-METHYLEN3-OXABICYCLO[3.3.0]OCTAN - 2-ONE & 21.37 & 2.21 & 72 \\
\hline 9 & O-Menthan-8-ol & 21.56 & 1.64 & 47 \\
\hline 10 & 1-methylbicyclo[2.2.1]hepta-2-ene & 21.84 & 6.12 & 49 \\
\hline 11 & (1RS,2RS,4RS)-2-Propylbicyclo[2.2. 2]oct-5-en-2-ol & 22.76 & 6.44 & 64 \\
\hline 12 & 1-methoxy-1,3-cyclohexadiene & 22.94 & 5.56 & 78 \\
\hline 13 & 2,3,5-Trimethylfuran & 23.46 & 0.81 & 35 \\
\hline 14 & 1-methoxy-1,3-cyclohexadiene & 23.69 & 5.79 & 72 \\
\hline 15 & Cyclopentanone, 3-methyl-2-(2-pent 68016 enyl)- & 23.74 & 2.65 & 27 \\
\hline 16 & Furan, 2,3-dihydro-4-methyl & 24.74 & 2.52 & 27 \\
\hline 17 & 1,4-Pentadiene, 2,3,3-trimethyl & 25.21 & 1.99 & 38 \\
\hline 18 & 2,3,3-Trimethyl-1-hexene & 25.61 & 7.03 & 27 \\
\hline 19 & 1-Pentanone, 1-(3-furanyl)-4-hydro xy & 25.88 & 5.51 & 43 \\
\hline 20 & hydroxy citronellal & 26.18 & 1.28 & 38 \\
\hline 21 & Cyclohexanol, 1-methyl-4-(1-methyl ethyl) & 26.95 & 5.83 & 47 \\
\hline 22 & $(1 \mathrm{R}, 2 \mathrm{~S})$-2-acetyl-1-methylcyclobuta neacetic acid & 27.52 & 1.77 & 22 \\
\hline 23 & 2-Cyclopentene-1-thione, 3-methyl & 29.47 & 3.92 & 58 \\
\hline 24 & $\begin{array}{l}\text { Bicyclo[3.1.1]heptane, 2,6,6-trime thyl-, [1R- } \\
\text { (1.alpha.,2.alpha.,5.al pha.)]- }\end{array}$ & 30.61 & 5.70 & 38 \\
\hline Total & & & 99.87 & \\
\hline
\end{tabular}




\section{Discussion}

Antibiotic resistance, as a critical public health concern, has accelerated research into antimicrobials of natural origin. Therefore, new antimicrobials are needed to control these pathogens, whose resistance is increasing. A number of studies have been conducted in recent years to detect bioactive compounds of plant origin in the hope of controlling antibiotic-resistant bacteria. Herbs have been an important source of medicine and alternative medicine to combat disease from ancient times [37]. Of the over 500,000 plant species known and unidentified, only $1-10 \%$ are used as animal and human foods [38, 39]. Interestingly, about $50 \%$ of the medicinal products used in the United States are of plant origin [39].

In the present study, the antimicrobial effects of plant materials tested were different on distinct $E$. coli isolates. Numerous factors are involved in this difference in efficacy, including plant cultivar, environmental conditions affecting plant growth, extracting components, the type of solvent used for extraction and resistance of isolates [40]. The MIC and MBC, as well as the mean diameter of the growth inhibition zone caused by ethanolic and aqueous extracts of Eryngium caucasicum Trautv were different from other studies, although most of the antimicrobial studies have been based on the essential oil of this plant $[40,41]$

In a report published by Thiem et al. [42], the antimicrobial activity of leaves and roots ethanolic extracts of three species of Eryngium, including Eryngium planum, Eryngium campestre and Eryngium maritimum, was evaluated against gram-positive Staphylococcus aureus and Bacillus subtilis. Their findings showed that the leaves extract effect on $S$. aureus was greater than $B$. subtilis while the root extract effect was the reverse. Comparison with our study, the MIC range of extracts and essential oil was $11.11-0.011 \mathrm{mg} / \mathrm{ml}$ on $E$. coli isolates, the MIC spectrum of leaves and root extracts on $S$. aureus and B. subtilis was 0.4$1.9 \mathrm{mg} / \mathrm{ml}$ and $1.3-5 \mathrm{mg} / \mathrm{ml}$, respectively [42]. In another study, the activity of methanolic, n-hexane, chloroform, ethyl acetate and aqueous Eryngium caeruleum extracts were assayed on Enterococcus faecalis, E. coli (UTI), Proteus mirabilis (UTI), Salmonella typhi, Klebsiella pneumoniae (UTI) and Pseudomonas aeruginosa. The results of this report on $E$. coli showed that the aqueous extract had the largest growth inhibition zone diameter, but the lowest MIC was associated with the n-hexane extract. On the other hand, their results for other bacteria were completely different [43]. The n-hexane, dichloromethane and methanol extracts from three species of Eryngium, including Eryngium caerulum, Eryngium thyrsoideum and Eryngium thyrsoideum, did not exhibit antibacterial activity on E coli in Karimi et al. [44], research.

The antibacterial activity of the leave and stem methanolic extracts of Eryngium serbicum L. was reported to be low against $E$. coli, but the flowers and roots extracts showed remarkable antibacterial activity. In contrast to our study that chlorogenic acid was not detectable, this compound was the most abundant compound of flower and stems extracts of Eryngium serbicum, and the amount of quercetin in 
the extracts of different parts of the plant was also higher than the amount of quercetin measured in the Eryngium caucasicum Trautv extracts tested in present study [45].

Menchavez et al. [46] findings from the study of the effect of leaves ethanolic extract of Eryngium foetidum on E. coli, as well as the extract components that may be involved in their antimicrobial function, showed that the extract had a mean diameter of growth inhibition zone $20 \mathrm{~mm}$ on $E$. coli culture. The extract also contains compounds including alkaloids, tannins, amines, unsaturated steroids quaternary, bases, 2-doxysugars and, contrary to our study, lack flavonoids and saponins [46].

Among the antimicrobial compounds tested in this survey, catechin and quercetin were detected in both types of Eryngium caucasicum Trautv extracts, which were higher in aqueous extract than ethanolic extract, although the antimicrobial effect of ethanolic extract was generally higher than that of aqueous extract. This is probably because of other antimicrobial compounds in ethanolic extracts or more potent antibacterial compounds. The antibacterial mechanism of catechins is the effect on cell wall and membrane as well as the production of hydrogen peroxide [47, 48], while quercetins inhibit DNA gyraze [49]. There are no reports of catechin and quercetin in extracts of this species of Eryngium caucasicum Trautv. However, Paun et al. [50] identify quercetin and chlorogenic acid in the ethanolic extract of Eryngium planum [50], and Marcetik et al. [51] detect catechin and chlorogenic acid in the root methanolic extract of E. palmatum [51]. In comparison to their reports, both flavonoids including catechin and quercetin were higher in aqueous extract than in ethanolic extract in our study. These compounds have a phenolic structure and are soluble in water and ethanol and are easily extracted using them, but are probably more soluble in aqueous extracts than ethanolic extracts and maybe that is why they abound more in aqueous extract.

According to reports confirming the existence of Froriepia subpinnata species in Asia, Africa and Europe, very little research has been conducted on extracts and essential oils of this plant and their antimicrobial effects in these continents [25].

The Froriepia subpinnata ethanolic extract, which was the most effective antimicrobial product after the plant's essential oil, was capable of inhibiting and killing $43 \%$ and $20 \%$ of all isolates, respectively. Two antimicrobial compounds including catechin and quercetin have been identified in this product, although there are no reports of these compounds in this plant, but Jorkesh et al. [52], and Ebrahimzadeh et al. [53], exhibit the presence of flavonoids and phenolic compounds as well as chlorogenic acid in methanolicacetic acid extract, and the flavonoids and phenol in aqueous extracts of Froriepia subpinnata, respectively.

Froriepia subpinnata essential oil, which was introduced as the most potent herbal product, was able to inhibit and kill $54 \%$ and $34 \%$ all isolates at concentrations of total isolates. The inhibitory effects of this product were higher compared to 14 antibiotics with the exception of meropenem, imipenem, piperacillin, chloramphenicol, cephalothin and cefepime antibiotics $(P<0 / 05)$. Most of the compounds in the essential oil of this plant, including terpenes, alcohols and aromatic compounds have been shown to have antibacterial effects in other studies $[54,40]$. It may be because of the diversity of these 
antimicrobial molecules in the essential oil that their antimicrobial effects are greater than those of ethanolic and aqueous extracts.

In the research of Mohammadzadeh et al. [55] which was performed on Froriepia subpinnta essential oil isolated from the Guilan region, the inhibitory and killing effects of this product on $P$. aeruginosa strain was less than the inhibitory and killing effect of this product on $E$. coli isolates used in this study. Although the plant species, the collection area and the essential oil extraction method of Froriepia subpinnta were almost similar in our study and their research, the antibacterial activity and the essential oil components in both studies are different. However, in the present study, the essential oil yield (the essential oil volume to dry weight ratio) was $0.26 \%$ and this value was almost similar to their study. Essential oil components vary based on factors such as plant cultivar, plant chemotype, harvesting time and climatic condition $[56,57,58]$.

In comparison to our research, $79.8 \%$ of Froriepia subpinnta essential oil collected from Mazandaran region, Iran, was composed of monoterpenoids in the report of Semnani et al. [59]. Another research was carried out by Mirzania et al. [60], on the components of Froriepia subpinnta essential oil and its antimicrobial activity from Mazandaran region, similar to the Semnani research [59], most of the essential oil was composed of monoterpene. The MIC and MBC values of effective essential oil on gramnegative bacteria including $E$. coli, Enterobacter faecalis and $P$. aeruginosa as well as gram-positive bacterium such as $S$. aureus was lower than the MIC and MBC values of Froriepia subpinnta essential oil used in the current study.

\section{Conclusion}

Therefore, by comparing the results of the present study with other studies, it can be expected that the components of plant products as well as their antimicrobial activity would be different under environmental conditions, plant cultivars, solvent type used for extracting extracts and isolate type. The antibacterial activity of extracts, essential oils and their chemical composition can be a valuable source for the development of new antibiotics. Although water solubility, structural diversity, molecular weight and hydrogen-based capacity of essential oil components can be affect their antibacterial potential. Since the antimicrobial effects of the natural products examined, of which Froriepia subpinnta essential oil had more antimicrobial potency and was even more effective than most of the antibiotics used, these products could be candidates for treatment of UTIs caused by E coli.

\section{Declarations}

\section{Acknowledgements}

The author sincerely thanks the faculty of sciences, University of Guilan, for providing laboratory facilities.

\section{Author details}


${ }^{1}$ University of Guilan, University Campus 2, Rasht, Iran. ${ }^{2}$ Department of Biology, Faculty of Sciences, University of Guilan, Rasht, Iran.

\section{Funding}

None.

\section{Availability of data and materials}

That all data and materials are available and included in the article.

\section{Ethics approval and consent to participate}

That ethics approval is not applicable.

\section{Consent to participate}

That consent participates is not applicable.

\section{Competing interests}

The authors declare no competing interests.

\section{Authors' contributions}

This work was taken from the thesis of MA. The idea, data analysis and conduction of this research were made by MJMM, while the experimental steps were performed by MA. This paper has been interpreted and written by MJMM.

\section{References}

1. Kucheria R, Dasgupta P, Sacks SH, Khan MS, Sheerin NS. Urinary tract infections: new insights into a common problem. Postgrad Med J. 2005;81(952):83-6.

2. Stamm WE, Norrby SR. Urinary tract infections: disease panorama and challenges. J Infect Dis. 2001;183(1):S1-4.

3. Russo TA, Johnson JR. Medical and economic impact of extraintestinal infections due to Escherichia coli: focus on an increasingly important endemic problem. Microbes Infect. 2003;5 (5):449-56.

4. National policy on traditional medicine and regulation of herbal medicines: Report of a WHO global survey. Switzerland: World Health Organization,Geneva; 2005.

5. Chandra H, Bishnoi P, Yadav A, Patni B, Mishra AP, Nautiyal AR. Antimicrobial resistance and the alternative resources with special emphasis on plant-based antimicrobials. Plants. 2017;6(2):1-16.

6. Dan MM, Sarmah P, Vana DR, Dattatreya A. Wound Healing: Concepts and updates in herbal medicine. Int J Med Res Health Sci. 2018;7(1):170-81. 
7. Daglia M. Polyphenols as antimicrobial agents. Curr Opin Biotechnol. 2012;23(2):74-181.

8. Tunçtürk M, Özgökçe F. Chemical composition of some Apiaceae plants commonly used in herby cheese in eastern Anatolia. Turk J Agric For. 2015;39:55-62.

9. Wang P, Su Z, Yuan W, Deng G, Li S. Phytochemical constituents and pharmacological activities of Eryngium L. (Apiaceae). Pharmaceutical Crops. 2012;3.99-120.

10. Zhang ZZ, Li SY, Ownby S, Wang P, Yuan W, Zhang WL et al. Phenolic compounds and rare polyhydroxylated triterpenoid saponins from Eryngium yuccifolium. Phytochemistry. 2008;69:207080.

11. Facciola S. Cornucopia: A Source book of edible plants. California; Kampong publications; 1990.

12. Ekpong B, Sukprakarn S. Harvest stages and umbel order contribution on eryngo (Eryngium foetidum L.) seed yield and quality. Kasetsart J. 2006;40:273-79.

13. Khoshbakht K, Hammer K, Pistrick K. Eryngium caucasicum Trautv cultivated as a vegetable in the Elburz Mountains (Northern Iran). Genet Resour Crop Evol. 2006;54:445-48.

14. Redzic SS. The ecological aspect of ethnobotany and ethnopharmacology of population in Bosnia and Herzegovina. Coll Antropol. 2007;31(3):869-90.

15. Abu-Rabia A. Herbs as a food and medicine source in Palestine. Asian Pac J Cancer Prev. 2005;6:404-7.

16. Kupeli E, Kartal M, Aslan S, Yesilada E. Comparative evaluation of the anti-inflammatory and antinociceptive activity of Turkish Eryngium species. J Ethnopharmacol. 2006;107:32-7.

17. Ayoub NA, Kubeczka KH, Nawwar MAM. An unique n-propyl sesquiterpene from Eryngium creticumL. (Apiaceae). Pharmazie. 2003;58:674-76.

18. Abbaspour Z, Jaimand K, Mozaffari S (2015) Comparison of essential oils compositions of Eryngo (Eryngium caucasicum) in different parts of plant in two growth conditions. J Med Plants Byproducts 1:83-98.

19. Palá-Paúl J, Copeland LM, Brophy JJ, Goldsack RJ. Essential oil composition of Eryngium paludosum (Moore et Betche) P. W. Michael: An endemic species from Eastern Australia. J Essential Oil Res. 2008;20(5):416-19.

20. Muckensturm B, Boulanger A, Farahi M, Reduron JP. Secondary metabolites from Eryngium species. Nat Prod Res. 2010;24(5):391-97.

21. Mozaffarian V. Umbelliferae. Flora of Iran, Research Institute of Forests and Rangelands, Tehran; 2007.

22. Rechinger KH. Flora Iranica. Graz: Akademische Druck-U, Verlagsanstalt; 1987.

23. Jodral MM. Illicium, Pimpinella and Foeniculum (Medicinal and Aromatic Plants), CRC Press;2004.

24. Seyedabadi MM, Amirabadi AA, Taheri A, Kashani Nejad M. The effect of infrared drying on the drying kinetics and leaf color index of Froriepia subpinnata. Faslnam Fanavari Novin Ghaza. 2016;13:45-7. 
25. Gülçın I, Oktay M, Kıreçcı E, Küfrevıoğlu Ö.i . Screening of antioxidant and antimicrobial activities of anise (Pimpinella anisum L.) seed extracts. J. Food Chem. 2003;83(3):371-82.

26. Cheesbrough M. Manual of Medical Microbiology. Low price ed. Oxford Press; 2000.

27. Clinical and Laboratory Standards Institute (CLSI). Performance standards for antimicrobial susceptibility testing: Twenty-first information supplement M100-S21. CLSI, Wayne. 2011.

28. Kumar PPNV, Shameem U, Kollu P, Kalyani RL, Pammi SVN. Green synthesis of copper oxide nanoparticles using Aloevera leaf extract and its antibacterial activity against fish bacterial pathogens. BioNanoScience. 2015;5(3):135-39.

29. Weseler A, Geiss HK, Saller R, Reichling J. A novel colorimetric broth microdilution method to determine the minimum inhibitory concentration (MIC) of antibiotics and essential oils against Helicobacter pylori. Pharmazie. 2005;60:497-502.

30. Volokhov D, Chizhikov V, Chumakov K, Rasooly A. Microarray analysis of erythromycin resistance determinants. J Appl Microbial. 2003;95(4):787-98.

31. Council of Europe, Strasbourg Cedex. European Pharmacopoeia. 5 th ed. France, 2.8.12; 2004.

32. NCCLS. Performance Standards for Antimicrobial Disc Suspectibility Tests. Villanova, PA: Approved Standard NCCLS Publication M2-A5;1993.

33. Ginovyan M, Petrosyan M, Trchounian A. Antimicrobial activity of some plant materials used in Armenian traditional medicine. BMC Complemen Altern Med. 2017;17:50-9.

34. Bhattacharjee S, Aditya M, Sil T. RP-HPLC and GC-MS based identification of phenolic acids, flavonoids and hydroxyl containing compounds from one of the lead accessions of Amaranthus hypochondriacus $\mathrm{L}$. identified on the basis of biomarkers of antioxidant potential. Basic Appl Pharm Pharm. 2018;(1.1):BAPP-1-102.

35. Adams RP. Identification of essential oil components by gas chromatography/quadropole mass spectroscopy. Allured Publishing Crop, Carol Stream, IL; 2007.

36. Ashoura ML, El-Readia M, Younsb M, Mulyaningsiha S, Sporera F, Efferthc T et al. Chemical composition and biological activity of the essential oil obtained from Bupleurum marginatum (Apiaceae). J Pharm Pharmacol. 2009;61(8):1079-87.

37. Subramani R, Narayanasamy M, Feussner KD. Plant-derived antimicrobials to fight against multidrug-resistant human pathogens. 3 Biotech. 2017;7:172.

38. Borris RP. Natural products research: perspectives from a major pharmaceutical company. $J$ Ethnopharmacol. 1996;51:29-38.

39. Cowan MM. Plant products as antimicrobial agents. Clin Microbiol Rev. 1999;12:564-82.

40. Hamedi A, Pasdaran A, Pasdaran A. Antimicrobial activity and analysis of the essential oils of selected endemic edible Apiaceae plants root from Caspian Hyrcanian region (North of Iran). Pharm Sci. 2019;25(2):138-44.

41. Abbaspour Z, Jaimand K, Mozaffari S. Comparison of essential oils compositions of Eryngo (Eryngium caucasicum) in different parts of plant in two growth conditions. J Med Plants By- 
products. 2015;1:83-98.

42. Thiem B, Goślińska O, Kikowska M, Budzianowski J. Antimicrobial activity of three Eryngium L. species (Apiaceae). Herba Pol. 2010;56(4):52-9.

43. Sadiq A, Ahmad S, Ali S, Ahmad F, Ahmad S, Zeb A et al. Antibacterial and antifungal potentials of the solvents extracts from Eryngium caeruleum, Notholirion thomsonianum and Allium consanguineum. BMC Complement Altern Med. 2016;16:478.

44. Karimi S, Lotfipour F, Asnaashari S, Asgharian P, Sarvari Y, Hazrati S. Phytochemical Analysis and Anti-microbial Activity of Some Important Medicinal Plants from North-west of Iran. Iran J Pharm Res. 2019;18(4):1871-83.

45. Vukic MD, Vukovic NL, Djelic GT, Obradovic A, Kacaniova MM, Markovic S et al. Phytochemical analysis, antioxidant, antibacterial and cytotoxic activity of different plant organs of Eryngium serbicum L. Ind Crop Prod. 2018;115:88-97.

46. Menchavez MY, Catipay JD, Espra AS, Castillo PM. Antibacterial properties of Bambusa vulgaris (Bamboo) leaves and Eryngium foetidum (Culantro) leaves against Staphylococcus aureus and Escherichia coli bacteria. Global J Med Plant Res. 2018;6(1):6-13.

47. Bernal P, Zloh M, Taylor PW. Disruption of D-alanyl esterification of Staphylococcus aureus cell wall teichoic acid by the b-lactam resistance modifier ( $(\mathbb{Z})$-epicatechin gallate. J Antimicrob Chemother. 2009;63:1156e1162.

48. Akagawa M, Shigemitsu T, Suyama K. Production of hydrogen peroxide by polyphenols and polyphenol-rich beverages under quasi-physiological conditions. Biosci Biotechnol Biochem. 2003;67(12):2632-40.

49. Tim Cushnie TP, Lamb AJ. Antimicrobial activity of flavonoids. Int J Antimicrob Agents. 2005;26(5):343-56.

50. Paun G, Neagu E, Moroeanu V, Albu C, Savin S, Lucian Radu G. Chemical and bioactivity evaluation of Eryngium planum and Cnicus benedictus polyphenolic-rich extracts. BioMed Res Int. 2019;Article ID 3692605.

51. Marčetić MD, Petrović SD, Milenković MT, Niketić MS. Composition, antimicrobial and antioxidant activity of the extracts of Eryngium palmatum Pančić and Vis. (Apiaceae). Cent EuR J Biol. 2014;9(2):149-55.

52. Jorkesh A, Hamidoghli Y, Olfati J, Samizadeh H, Bakhshi., Palá-Paúl J. Morphological and biochemical variability of Froriepia. Int J Veg Sci. 2019;26(3):262-74.

53. Ebrahimzadeh MA, Nabav SM. Mineral elements and antioxidant activity of three locally edible and medicinal plants in Iran. Asian J Chem. 2010;22(8):6257-66.

54. Kalemba D, Kunicka A. Antibacterial and antifungal properties of essential oils. Curr Med Chem. 2003;10(10):813-29.

55. Mohammadzadeh M, Mahmoudi R, Ghajarbeygi P. Evaluation of chemical composition and antibacterial properties of Froriepia subpinntaessential oils from Guilan region: Before and after flowering. J Essent Oil Bear Plants. 2018;21(4):1119-27. 
56. Bylaite E, Venskutonis RP, Roozen J P. Influence of harvesting time on the composition of volatile components in different anatomical parts of lovage (Levisticum officinale Koch.). J Agric Food Chem. 1998;46:3735-40.

57. Bylaite E, Venskutonis R, P. Roozen J, A. Posthumus, M. Composition of Essential Oil of Costmary [Balsamita major (L.) Desf.] at Different Growth Phases. J Agric Food Chem. 2000;48:2409-14.

58. Santos-Gomes PC, Fernandes-Ferreira M. Organ- and season-dependent variation in the essential oil composition of Salvia officinalis L. cultivated at two different sites. J Agric Food Chem. 2001;49:2908-16.

59. Morteza-Semnani K, Saeedi M, Akbarzadeh M. The essential oil composition of Froriepia subpinnata (Ledeb.) Baill., J Essent Oil Res 21. 2009;(2):127-28.

60. MirzaniaF, SarrafiY, Moridi Farimani M. Comparative evaluation of chemical compositions and biological activities of wild and cultivated Froriepia subpinnata L. Essent Oils. 2019;21 (2):331-40.

\section{Figures}

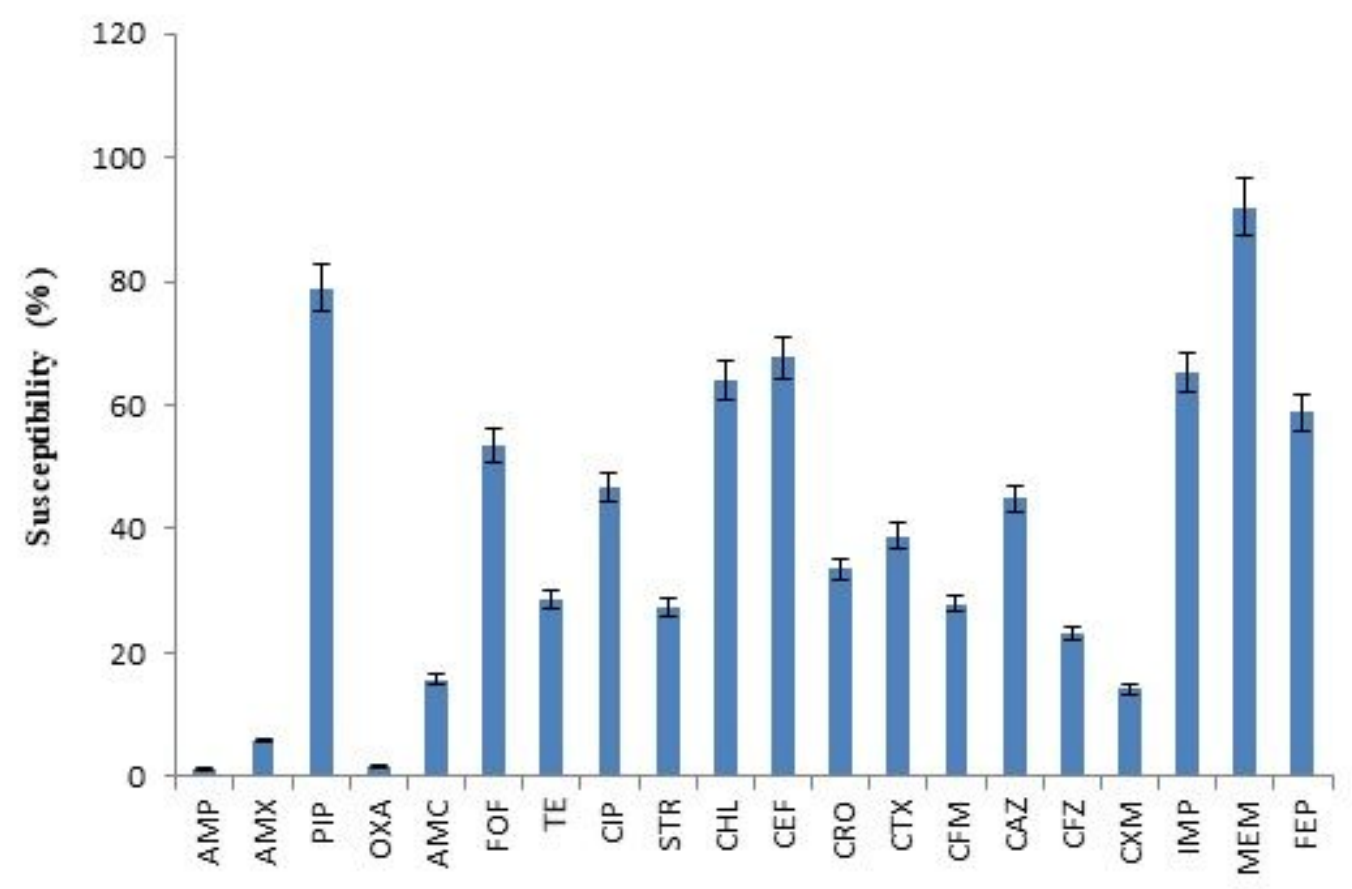

\section{Figure 1}

Susceptibility profile of clinical E. coli isolates to 20 different antibiotics. 


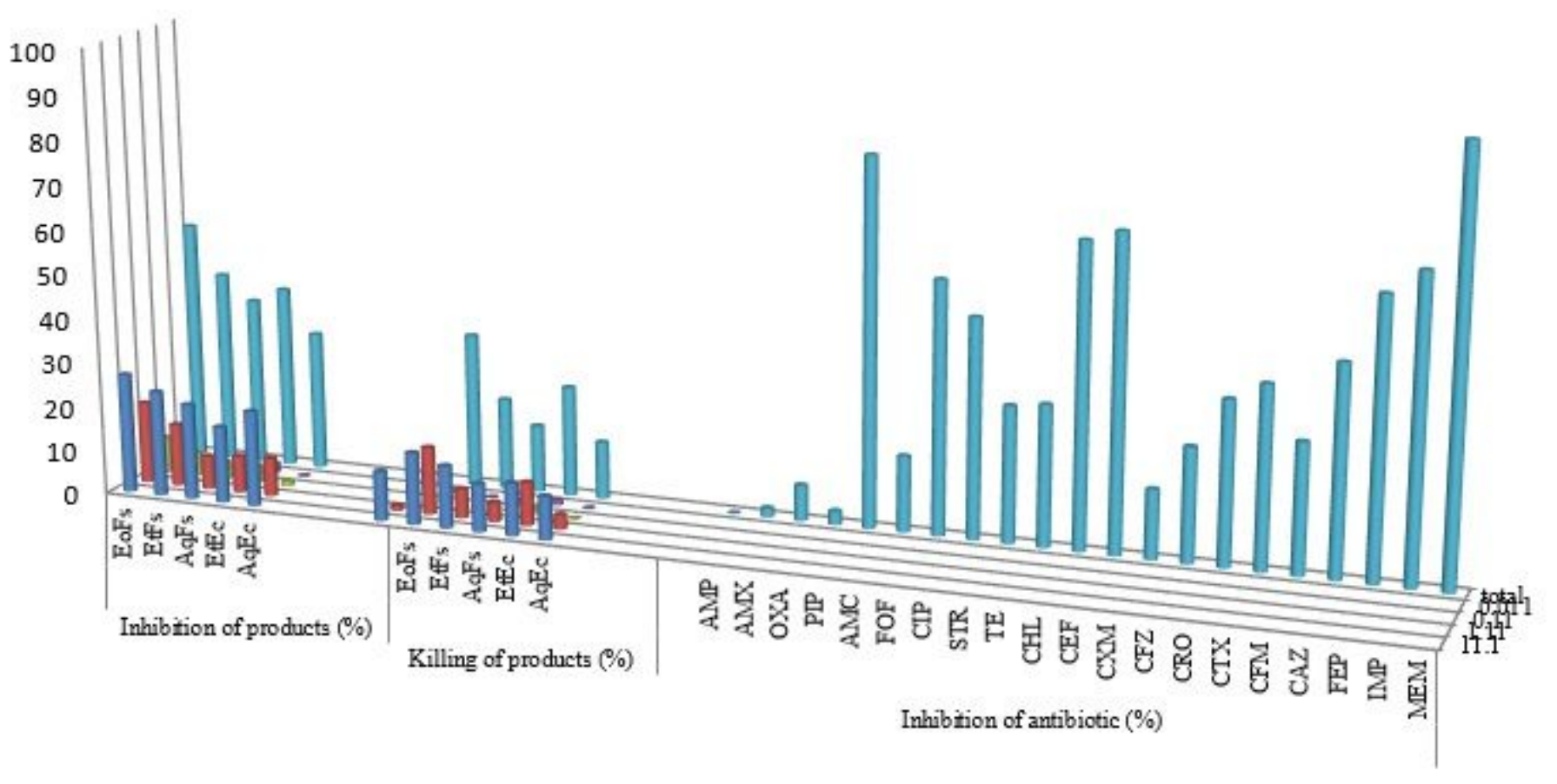

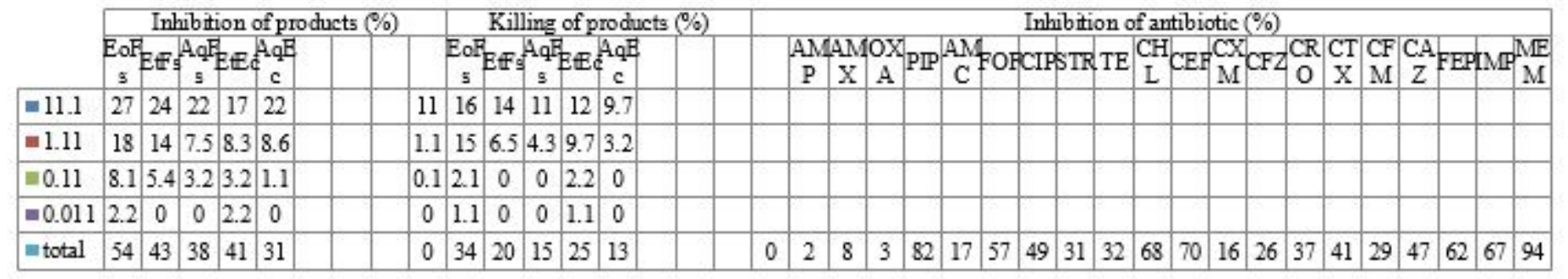

\section{Figure 2}

The inhibitory and killing effects (\%) of various concentrations $(11.11,1.11,0.11$ and $0.011 \mathrm{mg} / \mathrm{ml})$ of herbal products against on 91 MDR E. coil. EoFs: essential oil of Froriepia, EtFs: ethanolic extract of Froriepia subpinnata, AqFs: aqueous extract of Froriepia subpinnata, EtEc: ethanolic extract of Eryngium caucasicum Trautv, AqEc: aqueous extract of Eryngium caucasicum Trautv. 

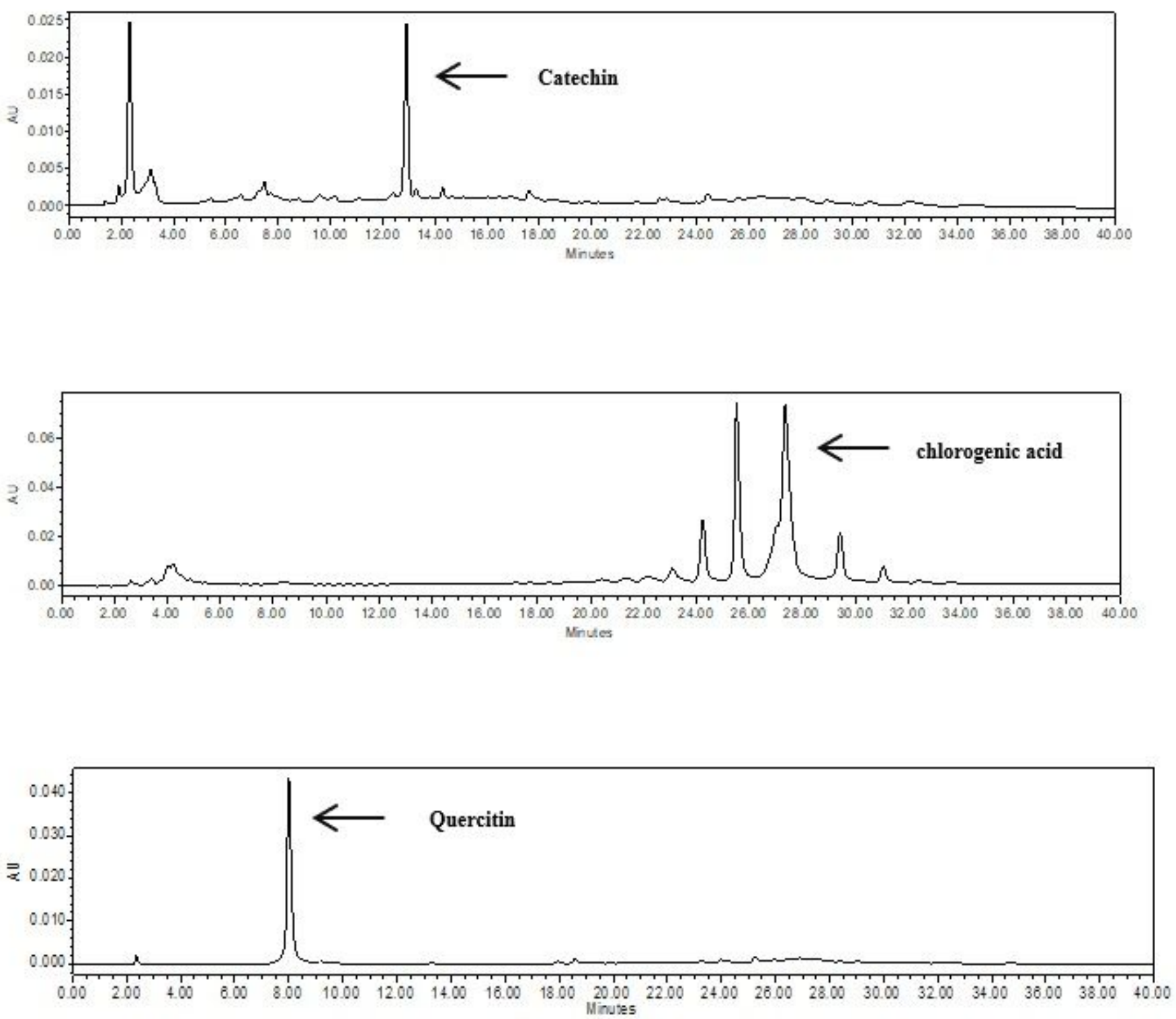

\section{Figure 3}

RP-HPLC chromatogram of the analytical standards for catechin, chlorogenic acid and quercetin at 280, 280 and $320 \mathrm{~nm}$, respectively. The arrows indicate the peaks of each of the above mentioned compounds. 

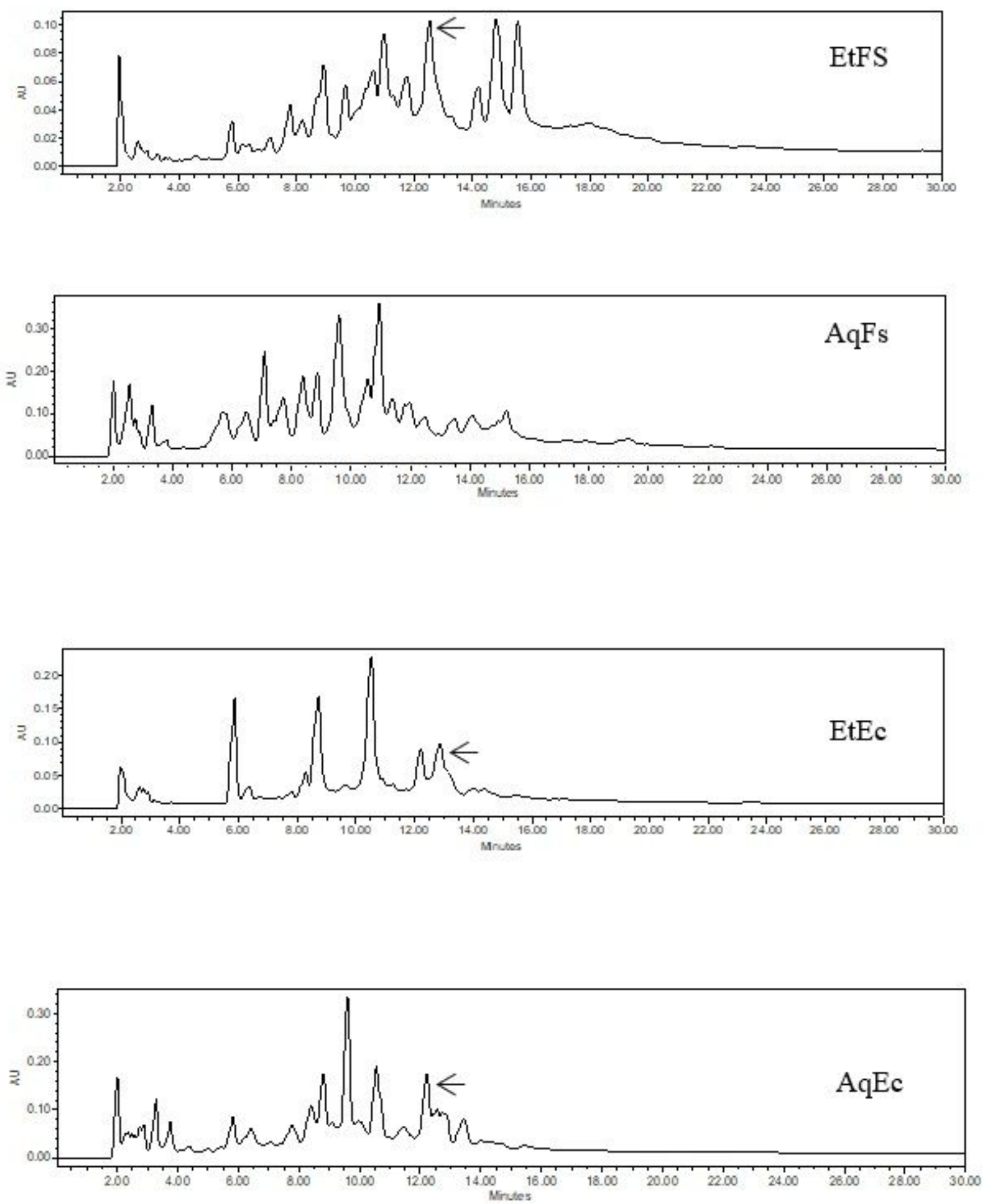

Figure 4

RP-HPLC chromatogram of EtFs, AqFs, EtEc and AqEc at $280 \mathrm{~nm}$. The arrow shows the peak of the catechin. Catechin was not detectable at AqEb. 

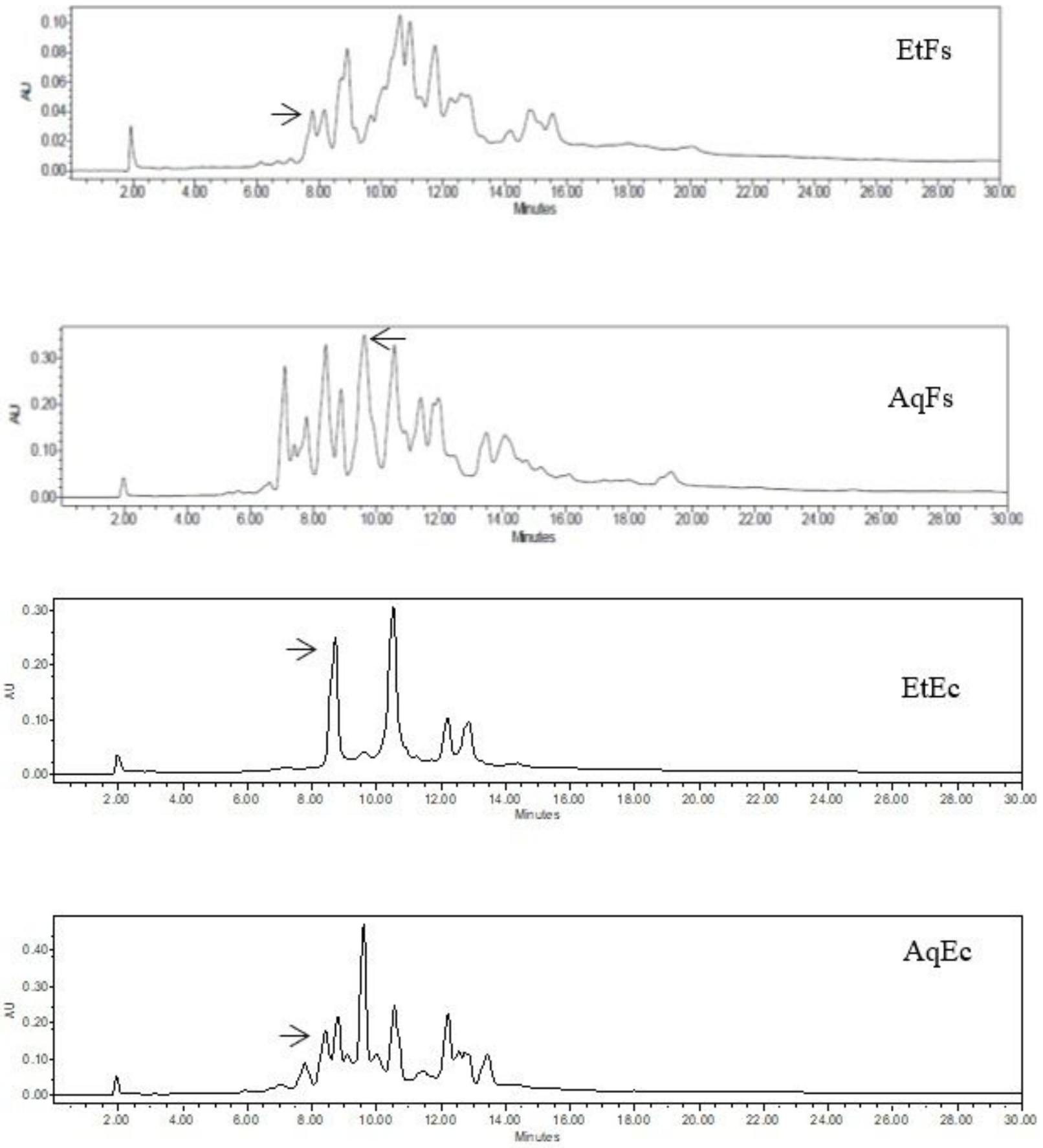

Figure 5

RP-HPLC chromatogram of EtFs, AqFs, EtEc and AqEc at $320 \mathrm{~nm}$. The arrow shows the peak of the quercetin. 


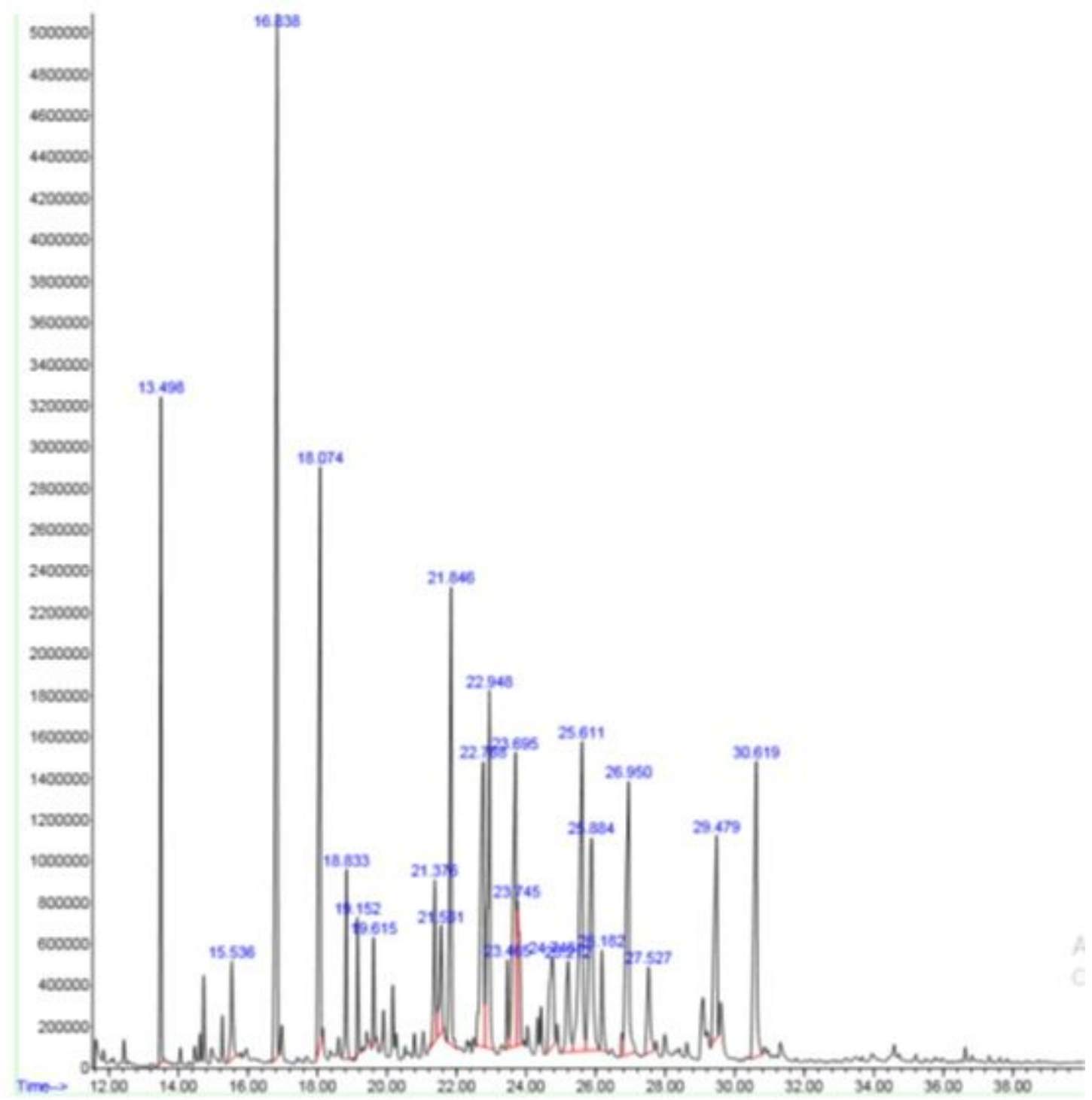

Figure 6

GC-mass chromatogram of EoFs. 